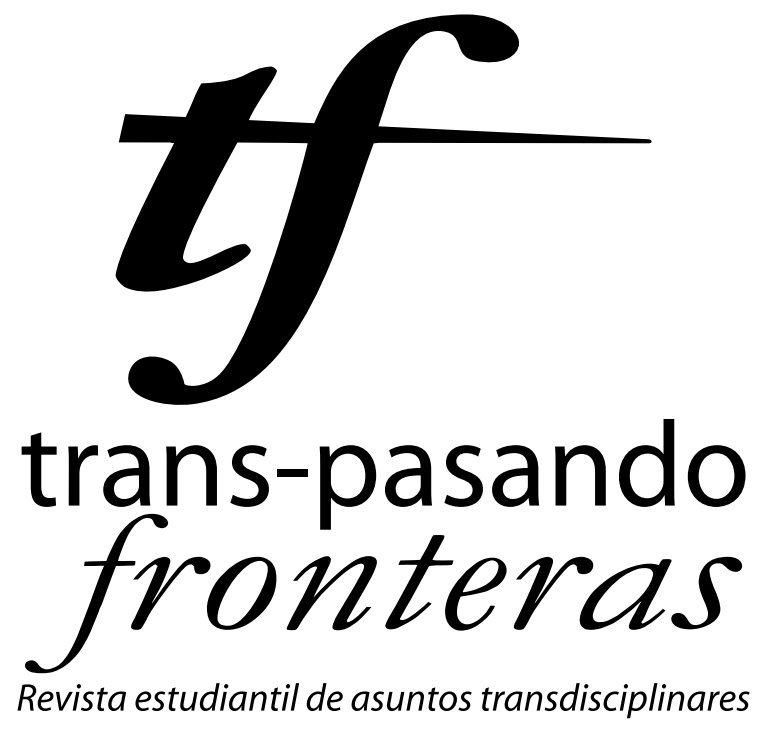

Una publicación de

Estudios

Interdisciplinarios

Jurídicos, Sociales

000 y Humanistas

ICESS

FACULTAD DE

DERECHO Y CIENCIAS

SOCIALES

然 UNIVERSIDAD 


\title{
La necesidad de la transdisciplinariedad en la educación superior ${ }^{*}$
}

\author{
Discurso central del Congreso internacional de educación \\ superior celebrado en Estambul, Turquía \\ 27-29 de mayo de 2011
}

Basarab Nicolescu, Prof.Dr. ${ }^{* *}$

\begin{abstract}
Resumen
Una educación viable solo puede ser una educación integral del ser humano. La educación transdisciplinar está fundada en la riqueza inagotable del espíritu científico, el cual se basa en el cuestionamiento y el rechazo de todas las respuestas a priori y en toda certeza contradictoria a los hechos. Al mismo tiempo, revaloriza el papel profundamente arraigado de la intuición, del imaginario, de la sensibilidad y del cuerpo en la transmisión de conocimiento. Es sólo de esta manera que la sociedad del siglo XXI puede reconciliar efectividad y respeto a la potencialidad de cada ser humano. El enfoque transdisciplinario será un complemento indispensable para el enfoque disciplinar, porque significa el surgimiento de seres continuamente conectados, capaces de adaptarse a las cambiantes exigencias de la vida profesional, dotados con una flexibilidad permanente orientada siempre hacia la actualización de sus potencialidades interiores. Si la universidad pretende ser un actor válido en el desarrollo sostenible, primero tiene que reconocer la aparición de un nuevo tipo de conocimiento: el conocimiento transdisciplinar. La nueva producción de conocimiento implica una apertura multidimensional necesaria en el proceso de aprender: hacia la sociedad civil; hacia el tiempo del ciberespacio; hacia el objetivo de la universalidad; hacia una redefinición de los valores que rigen su propia existencia.
\end{abstract}

* Traducción al español por Ana María Garay. Revisado y notas de píe de página por Adolfo A. Abadía. Título original The Need for Transdisciplinarity in Higher Education, tomado de http://basarabnicolescu.fr/Docs_articles/Istanbul_UIK2011.pdf

** Agradecimientos especiales al profesor Basarab Nicolescu, director del Centro Internacional de Investigaciones y Estudios Transdisciplinarios (CIRET) de Francia, por permitirnos publicar unas cuantas líneas sobre la transdisciplinariedad. 
Palabras claves:

Educación superior, transdisciplinariedad, niveles de realidad, lógica del tercero incluido, epistemología.

\section{Multi-inter y transdisciplinariedad}

El aumento sin precedentes del conocimiento en nuestra era plantea la pregunta desafiante de cómo adaptar nuestra mentalidad de ser, cómo decidir en una situación determinada de la vida cuando hoy hay más de 8.000 disciplinas, cómo adaptarse a los rápidos cambios en el conocimiento, lo que requiere cambiar de trabajo una o incluso varias veces durante la vida del ser humano si queremos enfrentar al desempleo. La universidad es uno de los lugares centrales para superar estos desafíos.

La armonía entre el ser interior y el conocimiento externo presupone que esos hechos conocidos serian inteligibles, comprensibles. Pero ¿puede existir tal comprensión en la era de la gran explosión disciplinaria y la especialización implacable?

La indispensable necesidad de puentes entre disciplinas diferentes se atestigua por la aparición de la multidisciplinariedad y la interdisciplinariedad a mediados del siglo XX.

Las preocupaciones multidisciplinares estudian un tema de investigación no solo en una disciplina sino en varias al mismo tiempo. Por ejemplo, La Mezquita $\mathrm{Azul}^{2}$ se puede estudiar no sólo dentro del contexto de la historia del arte, sino también dentro del contexto de la historia de las religiones, historia europea, o geometría. El tema en cuestión será en última instancia enriquecido al incorporar las perspectivas de varias disciplinas. El enfoque multidisciplinar desborda las fronteras disciplinares mientras su objetivo se limita al marco de la investigación disciplinaria.

La interdisciplinariedad tiene un objetivo diferente a la multidisciplinariedad. Se refiere a la transferencia de métodos de una disciplina a la otra. Por ejemplo, una transfiere los métodos de la física nuclear a la medicina, y esto lleva a la aparición de nuevos tratamientos para el cáncer. Al igual que la multidisciplinariedad, la interdisciplinariedad desborda las disciplinas, pero su objetivo sigue estando dentro del marco de la investigación disciplinaria.

Como indica el prefijo Trans, la transdisciplinariedad se preocupa por aquello

2 La Mezquita Azul fue construida entre 1609 y 1616 en Estambul y es considerada una de las principales mezquitas de la capital de Turquía. Lleva ese nombre a razón de la tonalidad de los azulejos que decoran su interior. 
que está entre las disciplinas, a través de ellas y más allá de toda disciplina. $\mathrm{Su}$ objetivo es la comprensión del mundo actual, en el cual uno de los imperativos es la unidad del conocimiento. La palabra en sí es bastante reciente: fue introducida por primera vez por Jean Piaget en 1970 (Piaget, 1970).

En la presencia de varios "niveles de Realidad", el espacio entre disciplinas y más allá de éstas está lleno, del mismo modo que el vacío cuántico está lleno de todas las potencialidades: desde la partícula cuántica hasta las galaxias, desde el Quark hasta los elementos pesados que condicionan la aparición de la vida en el universo.

La investigación transdisciplinar es claramente distinta de la investigación disciplinar, aun cuando son totalmente complementarias. La investigación disciplinar se preocupa, a lo sumo, por uno y el mismo nivel de Realidad; además, en la mayoría de los casos, solo se interesa por fragmentos de un nivel de Realidad. En contraste, la transdisciplinariedad se preocupa por las dinámicas engendradas por la acción de varios niveles de Realidad al mismo tiempo. El descubrimiento de estas dinámicas necesariamente pasa por el conocimiento disciplinar.

\section{Tabla 1}

Comparación entre educación disciplinar (ED) y educación transdisciplinar (ET)

\begin{tabular}{|c|c|}
\hline Educación Disciplinaria (ED) & Educación Transdisciplinar (ET) \\
\hline IN VITRO & IN VIVO \\
\hline Un nivel de Realidad & Varios niveles de Realidad \\
\hline Mundo externo - Objeto & $\begin{array}{c}\text { Correspondencia entre el mundo externo } \\
\text { (Objeto) y el mundo interno (Sujeto) }\end{array}$ \\
\hline Acumulación de conocimiento & Comprensión \\
\hline Inteligencia analítica & $\begin{array}{c}\text { Nuevo tipo de inteligencia - Armonía entre } \\
\text { mente, emociones y cuerpo }\end{array}$ \\
\hline Lógica Binaria & Lógica del tercero incluido \\
\hline (Verdad absoluta / Falsedad absoluta) & (Verdad relativa) \\
\hline Orientada hacia el poder y la posesión & Orientada hacia el asombro y compartir \\
\hline Exclusión de valores & Inclusión de valores \\
\hline
\end{tabular}


El conocimiento transdisciplinar corresponde a un conocimiento in vivo, preocupado por la reciprocidad entre el mundo externo del objeto y el mundo interno del sujeto. Por definición, el conocimiento transdisciplinar incluye un sistema de valores, valores humanistas. Esto lleva a un nuevo tipo de educación -la educación transdisciplinar (ET), distinta pero complementaria de nuestra actual educación disciplinar (ED).

Es importante tener en cuenta de que el conocimiento disciplinar y transdisciplinar no son antagónicos sino complementarios. Ambas metodologías están fundadas en la actitud científica. La construcción de la mente transdisciplinar en la universidad es nuestro principal desafío hoy.

Con el fin de explorar la nueva educación transdisciplinar, tenemos que entender lo que es la metodología transdisciplinar.

\section{La mirada transdisciplinar a la naturaleza y el conocimiento}

La metodología de la transdisciplinariedad se basa en tres postulados (Nicolescu, 2002):

1. El postulado ontológico: existen, en la naturaleza y en nuestro conocimiento de la naturaleza, diferentes niveles de la Realidad del objeto y diferentes niveles de la realidad del sujeto.

2. El postulado lógico: el paso de un nivel de Realidad a otro, está asegurado por la "lógica del tercero incluido".

3. El postulado epistemológico: la estructura de la totalidad de los niveles de Realidad es una estructura compleja: cada nivel es lo que es gracias a que todos existen al mismo tiempo.

Los primeros dos postulados recibieron, en el siglo XX, evidencia experimental de la física cuántica, mientras que el último tiene su origen no solo en la física cuántica sino también en una variedad de otras ciencias exactas y humanas.

El concepto clave del enfoque transdisciplinar de la naturaleza y el conocimiento es el concepto de niveles de Realidad.

Aquí está la definición que le damos a la palabra -realidad- la cual es pragmática y ontológica al mismo tiempo.

Por realidad (Nicolescu, 2009) pretendemos en primer lugar designar lo que resiste 
nuestras experiencias, representaciones, descripciones, imágenes, y hasta incluso formulaciones matemáticas.

En la medida en que la naturaleza participa en el ser del mundo, hay que asignar también una dimensión ontológica al concepto de Realidad. Realidad no es simplemente una construcción social, el consenso de una colectividad o un acuerdo intersubjetivo. También tiene una dimensión trans-subjetiva: por ejemplo, los datos experimentales pueden arruinar la teoría científica más hermosa.

Por supuesto, hay que distinguir las palabras "Real" y "Realidad". Real designa lo que es, mientras Realidad está conectada a la resistencia en nuestra experiencia humana. Lo "Real" es, por definición, oculto para siempre, mientras que la "Realidad" es accesible a nuestro conocimiento.

Por nivel de Realidad, una noción introducida por primera vez en 1985 (Nicolescu, 1985), designo a un conjunto de sistemas que son invariantes bajo ciertas leyes, por ejemplo, las entidades cuánticas están subordinadas a las leyes cuánticas, las cuales se apartan radicalmente de las leyes del mundo macrofísico. Esto es para decir que dos niveles de Realidad son diferentes si, mientras pasa de uno al otro, se produce una ruptura en las leyes aplicables y una ruptura en conceptos fundamentales (como por ejemplo, la casualidad).

La aparición de al menos tres niveles diferentes de Realidad en el estudio de los sistemas naturales -el nivel macrofísico, el nivel microfísico y el ciber-tiempoespacio (al cual se le podría agregar un cuarto nivel, el de la supercuerdas unificando todas las interacciones físicas) - es un gran acontecimiento en la historia del conocimiento.

Dos niveles adyacentes están conectados por la "lógica del tercero incluido", que surgió como una lógica cuántica válida.

Nuestra comprensión del axioma del tercero incluido -ahí existe un tercer término " $\mathrm{T}$ " el cual es al mismo tiempo "A" y "no-A"- está completamente aclarado una vez se introduce la noción de niveles de Realidad.

Con el fin de obtener una idea clara del significado del tercero incluido, podemos representar los tres términos de la nueva lógica - "A", "no-A" y "T" - y las dinámicas asociadas con ellos por un triángulo en el que uno de los vértices se 
sitúa en un nivel de Realidad y los otros dos vértices en otro nivel de Realidad. Si uno permanece en un solo nivel de Realidad, todos los fenómenos parecen ser el resultado de la lucha entre dos elementos contradictorios. La tercera dinámica, la del T-estado, se ejerce en otro nivel de Realidad, ahí donde había aparecido para ser separada es de hecho unida, y ahí donde había aparecido contradictoria, es percibido como no-contradictoria.

La acción transfinita de la lógica del tercero incluido en los diferentes niveles de Realidad induce una estructura Gödeliana de la unidad de los niveles de Realidad.

La estructura abierta de la unidad de los niveles de Realidad está de acuerdo con uno de los resultados científicos más importantes del siglo XX referentes a la aritmética, el teorema de Kurt Gödel ${ }^{3}$, el cual establece que un sistema de axiomas suficientemente rico inevitablemente conduce a resultados que no se deciden y son contradictorias. Las implicaciones del teorema de Gödel, tienen una importancia considerable para todas las teorías modernas del conocimiento, principalmente porque no solo concierne el campo de la aritmética, sino todas las matemáticas incluyendo la aritmética. La estructura Gödeliana de los niveles de Realidad implica la imposibilidad de una teoría completa cerrada en sí misma. El conocimiento siempre es abierto.

Un nuevo Principio de la Relatividad surge de la coexistencia entre la pluralidad compleja y la unidad abierta: Ningún nivel de Realidad constituye un lugar privilegiado desde el cual uno es capaz de entender todos los niveles de Realidad. Un nivel de Realidad es lo que es porque todos los otros niveles existentes al mismo tiempo. Este Principio de Relatividad es lo que origina una nueva perspectiva en la religión, la política, el arte, la educación, la vida social y, particularmente importante para nosotros en esta región del mundo, la construcción de Europa. Y cuando nuestra perspectiva en el mundo cambia, entonces, el mundo cambia.

No hay necesidad de inventar una universidad totalmente nueva, pero si hay que transformar las universidades disciplinares existentes mediante la adopción de la metodología transdisciplinar como su metodología complementaria.

3 Kurt Gödel, lógico estadounidense conocido por sus investigaciones en matemáticas y en filosofía. Es también conocido por lo que se conoce como el teorema de Gödel, el cual establece que en cualquier sistema (Realidad) simbólico formal es posible construir una proposición que no se puede probar ni refutar en el mismo sistema (Realidad). 
Pasos importantes en esta dirección se hicieron en varios países (CIRET, 2011). En particular, programas de doctorado transdisciplinares están establecidos ahora en Rumania y Sudáfrica.

El enfoque transdisciplinar es realista e incluso necesario para la supervivencia de las universidades contemporáneas, situadas en el contexto caótico de la globalización. Una condición necesaria es entender lo que la Realidad es hoy. Somos parte del movimiento ordenado de la Realidad. Nuestra libertad consiste en entrar en el movimiento o alterarlo. La Realidad depende de nosotros. La Realidad es plástica. Podemos responder al movimiento o imponer nuestra voluntad de poder y dominación. Nuestra responsabilidad es construir futuros sostenibles de acuerdo con el movimiento global de la realidad.

El surgimiento de una nueva cultura capaz de contribuir a la eliminación de las tensiones que amenazan la vida en nuestro planeta, será imposible sin un nuevo tipo de aprendizaje que tenga en cuenta todas las dimensiones del ser humano.

Una educación viable sólo puede ser una educación integral del ser humano. Esta, fundada en un nuevo tipo de inteligencia que requiere una armonía entre la mente, las emociones y el cuerpo. Los recientes hallazgos de la neurofisiología (Damasio, 2003) muestran claramente el rol excepcional de las emociones en la educación.

La educación transdisciplinar se funda en la inagotable riqueza del espíritu científico el cual se basa en el cuestionamiento, y la negativa de todas las respuestas a priori $\mathrm{y}$ toda certidumbre contradictoria a los hechos. Al mismo tiempo, revaloriza el papel de una intuición profundamente arraigada en el imaginario, en la sensibilidad y en el cuerpo a propósito de la transferencia de conocimiento. Es sólo de esta manera que la sociedad del siglo XXI puede reconciliar la efectividad y el respeto por el potencial de cada ser humano. El enfoque transdisciplinar será un complemento indispensable para el enfoque disciplinar, porque significará el surgimiento de seres conectados continuamente, quienes son capaces de adaptarse a las cambiantes exigencias de la vida profesional, y quienes están dotados con una flexibilidad permanente orientada siempre hacia la actualización de las potencialidades interiores.

El intercambio universal de conocimiento - una necesidad de nuestro mundo- no puede suceder sin el surgimiento de una nueva tolerancia fundada sobre la actitud transdisci- 
plinaria, que implique poner en práctica la visión transcultural. La actitud transcultural nos permite una mejor comprensión de nuestra propia cultura, una mejor defensa de nuestros intereses nacionales, y un mejor respeto de nuestras convicciones religiosas y políticas.

Si la Universidad pretende ser un actor válido en el desarrollo sostenible, tiene primero que reconocer el surgimiento de un nuevo tipo de conocimiento: el conocimiento transdisciplinar. La nueva producción de conocimiento implica una apertura multidimensional necesaria del proceso de aprender: hacia la sociedad civil; hacia el ciber-espacio-tiempo; hacia el objetivo de la universalidad; hacia una redefinición de los valores que gobiernan su propia existencia.

\section{Bibliografía}

CIRET (2011). Internet site of the International Center for Transdisciplinary Research. Consulta realizada el 26 de abril de 2011, desde: http://basarab.nicolescu.perso.sfr. fr/ciret/indexen.html

DAMASIO, Antonio (2003). Looking for Spinoza: Joy, Sorrow, and the Feeling Brain. San Diego, Harcourt.

NICOLESCU, Basarab. (1985). We, the Particle and the World / Nous, la particule et le monde. París, Le Mail. 2nd edition : 2002, Mónaco, Rocher.

- (2002). Manifesto of Transdisciplinarity. New York, State University of New York (SUNY) Press. Translation in English by Karen-Claire Voss. (2009). What is Reality ? / Qu'est-ce que la Réalité. Montréal, Liber.

PIAGET, J. (1970). "Epistemology of Interdisciplinary Relations / L'épistémologie des relations interdisciplinaires". In L'interdisciplinarité - Problèmes d'enseignement et de recherche dans les universités. París: OCDE. 